\title{
Preparation of samarium iron garnet nanoparticles via modified conventional mixing oxides method
}

\begin{abstract}
This work is concerned with the preparation of samarium iron garnet ( $\mathrm{Sm3Fe5O12)}$ nanoparticles via an improved technique named: Modified Conventional Mixing Oxides (MCMO) method. This material was characterized by XRD, FESEM, EDX and TEM. Metal oxides and ethanol solution were used as raw materials to prepare Sm3Fe5O12 (SmIG) material. Single-phase SmIG nanoparticles with an average particle value of $25 \mathrm{~nm}$ and average crystallite size value of $44 \mathrm{~nm}$ have been synthesized at $1350{ }^{\circ} \mathrm{C}$ via the MCMO method. SmIG powders with grain sizes below $1 \mathrm{\varepsilon m}$ and high purity have been presented by FESEM and EDX results, respectively. Lattice constant value of $12.535 \mathrm{~A}^{\circ}$ and density value of 6.221 g.cm-3, were calculated for the SmIG sample. The latter has reached around $99 \%$ of its theoretical density. The MCMO method appears to be an attractive route due to the enhancement of structural properties of the interested sample with high yield in the nanoscale product as compared to other preparation techniques.
\end{abstract}

Keyword: EDX; FESEM; MCMO method; SmIG; TEM; XRD 